\title{
On modelling of the buckling resistance of welded I-section columns
}

\author{
Radosław Szczerba ${ }^{1, *}$, Marcin Gajewski ${ }^{1}$, and Marian Giżejowski ${ }^{1}$ \\ ${ }^{1}$ Warsaw University of Technology, Faculty of Civil Engineering, Pl. Politechniki 1, 00-661 Warsaw, \\ Poland
}

\begin{abstract}
This paper analyzes the influence of geometrical and material imperfections on the buckling resistance of welded I-section columns subjected to axial compression through numerical and analytical models. The paper is divided into two parts. The first part recalls a FEM parametric study of members under compression taking into account different slenderness ratios, as well as different amplitudes of initial crookedness and different values of postwelding residual stresses. The formulation of analytical approach is the main issue of the second part of the paper. Analytical formulation of the buckling resistance is based on a statistical hypothesis of the minima value approach, called the Marchant-Rankine'sMurzewski approach (M-R-M). Calibration of imperfection factors included in the analytical formulation is made using the results of FEM simulations performed in the first stage of research investigations.
\end{abstract}

\section{Introduction}

Recent design strategies are based on advanced analysis applying the stiffness degradation concept to account for the combined effects of plasticity and imperfections on the nonlinear behaviour of structures. Many standards, including Eurocode 3 [1,2], permit the use of non-linear finite element method (FEM) simulations. Geometrically nonlinear refined plastic hinge analysis of GMNIA type (geometrically and materially nonlinear analysis with imperfections) is the method accurate enough in the resistance assessment for engineering practice.

The main aim of the paper is to assess the influence of geometrical and material imperfections on the buckling resistance of welded I-section columns subjected to axial compression. To achieve this goal the authors divided the paper into two parts. In the first part the results of a FEM parametric study (with the use of GMNIA) of compressed members were recalled [3]. The presented outcomes form the basis for the analytical formulation of the buckling resistance of welded I-section columns which is the subject of the second part of the paper. The analytical approach is based on a statistical hypothesis of the minima value approach $[4,5]$, called the Marchant-Rankine's-Murzewski approach (M$\mathrm{R}-\mathrm{M})$. Calibration of the imperfection factors contained in the analytical formulation is

\footnotetext{
*Corresponding author: r.szczerba@il.pw.edu.pl
} 
performed with the use of the results of FEM simulations recalled in the first stage of research investigations.

\section{Effect of I-section postwelding residual stresses on the equivalent stress-strain model}

Let us consider a perfectly straight column made of steel grade S355 with an elastic -plastic model of parent steel material, and with the same stress strain characteristics for tension and compression (Fig. 1a). A low value of the hardening modulus of $E_{\text {har }}=E / 1000$ is adopted. The initial self-equilibrated postwelding stresses distribution is approximated by adopting a standard residual stress block postulated in [3] (Fig. 1b). The buckling direction is about $z-z$ axis.

Table 1 presents the parameters $\alpha_{f} t_{f}$ and $\alpha_{w} t_{w}$ (the dimensions of respectively flange and web parts under tension) for the discrete values of $\psi_{\text {ten }}$ equal to 1.0 for the common steel grade S355 and $\psi_{\text {com }}$ from the range of $(0.1,0.5)$ with the interval of 0.1 .

a)

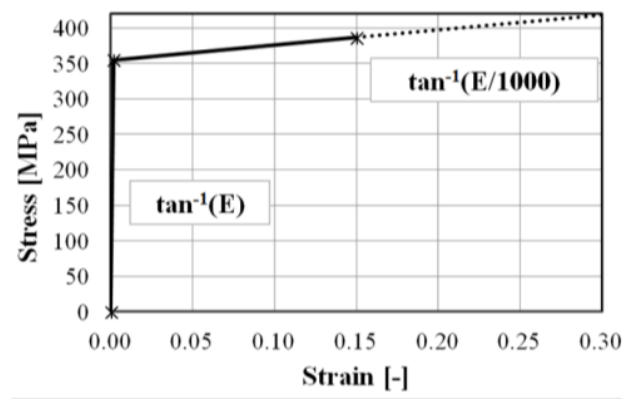

b)

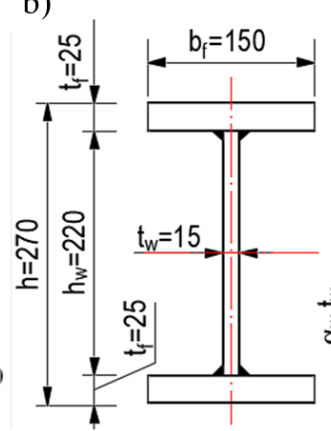

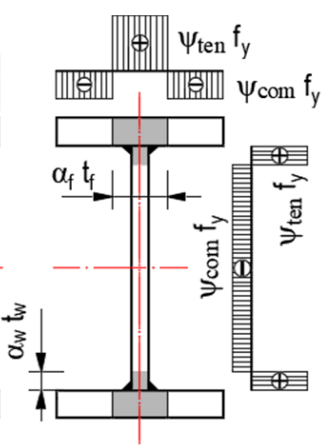

Fig. 1. Material characteristic and postwelding residual stresses distribution.

Table 1. The parameters describing the distribution of postwelding residual stresses [3].

\begin{tabular}{|l|l|l|l|l|l|}
\hline$\psi_{\text {ten }}$ & $\psi_{\text {com }}$ & $\begin{array}{l}\alpha_{f} t_{f} \\
{[\mathrm{~mm}]}\end{array}$ & $\begin{array}{l}\alpha_{w} t_{w} \\
{[\mathrm{~mm}]}\end{array}$ & $\begin{array}{l}\psi_{\text {ten }} \cdot f_{y} \\
{[\mathrm{MPa}]}\end{array}$ & $\begin{array}{l}\psi_{\text {com }} \cdot f_{y} \\
{[\mathrm{MPa}]}\end{array}$ \\
\hline 1.0 & $\mathbf{0 . 1}$ & 13.6 & 10.0 & 355.0 & $\mathbf{3 5 . 5}$ \\
\hline 1.0 & $\mathbf{0 . 2}$ & 25.0 & 18.3 & 355.0 & $\mathbf{7 1 . 0}$ \\
\hline 1.0 & $\mathbf{0 . 3}$ & 34.6 & 25.4 & 355.0 & $\mathbf{1 0 6 . 5}$ \\
\hline 1.0 & $\mathbf{0 . 4}$ & 42.9 & 31.4 & 355.0 & $\mathbf{1 4 2 . 0}$ \\
\hline 1.0 & $\mathbf{0 . 5}$ & 50.0 & 36.7 & 355.0 & $\mathbf{1 7 7 . 5}$ \\
\hline
\end{tabular}

When an I-section column is subjected to axial compression, firstly the column behaves elastically so that the first piece of multi-linear equivalent $\sigma_{\text {eff }}-\varepsilon_{\text {eff }}$ relationship is $\sigma_{\text {eff }}=E \varepsilon_{\text {eff }}$. The first yielding of compression zones starts when $N_{E d}=\left(1-\psi_{c o m}\right) N_{p l, R k}$ that corresponds to the reduced yield stress $\sigma_{y, \text { red }}=\left(1-\psi_{\text {com }}\right) f_{y}$. The column tangent stiffness is then suddenly degraded from its elastic value of $E I_{z}$ to its tangent value of $E_{T} I_{z}$.

The stress-strain relationship can be therefore formulated deterministically as a minimum of three variables being the functions of $\varepsilon$ :

$$
\sigma=\min \left(E \varepsilon, \quad \sigma_{H, e f f}+E_{T} \varepsilon, \quad \sigma_{y, \text { red }}+E_{\text {har }} \varepsilon\right)
$$


in which $\sigma_{H, e f f}$ is the value of initial postwelding stress (see Fig. 2a) and the initial stress value of rigid-plastic-strain-hardening part of the parent steel stress-strain relationship is expressed as:

$$
\sigma_{y, \text { red }}=\left(1-\frac{E_{h a r}}{E}\right) f_{y}
$$

The results of finite element simulations of the stub test representing the equivalent stress-strain model $\left(\sigma_{e f f}-\varepsilon_{e f f}\right.$ model $)$ for a considered steel grade of S355 are given in Fig. 2a, together with the estimates of $E_{T}$ and $\sigma_{H, \text { eff }}$ values $\left(\sigma_{H, \text { eff }, 0.1}\right.$ is for $\psi_{\text {com }}=0.1, \sigma_{H, \text { eff }, 0.2}$ is for $\psi_{\text {com }}=0.2$, etc.).
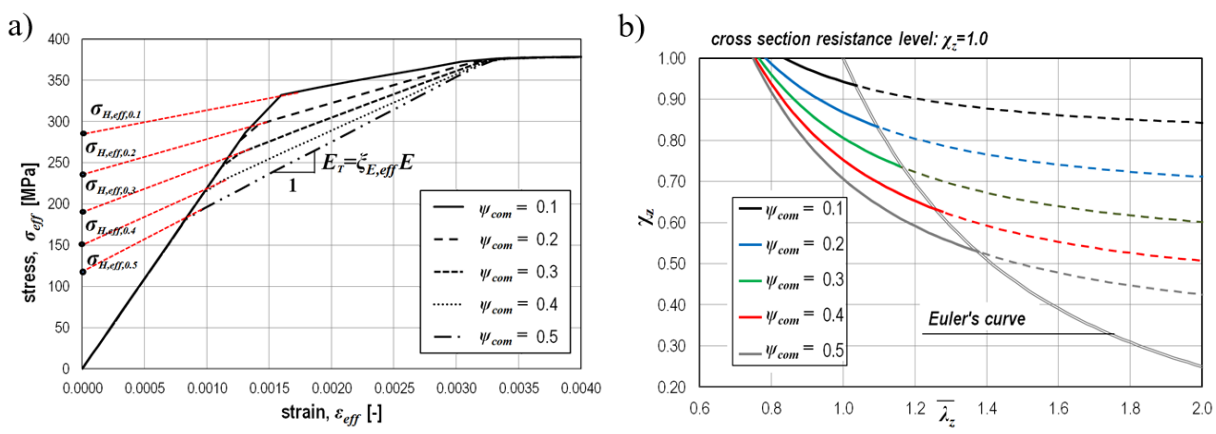

Fig. 2. Construction of equivalent stress-strain diagram for the post-welding steel (a), graphical interpretation of the buckling reduction factor (b).

The parameters of the equivalent stress-strain characteristic are given in Table 2.

Table 2. Parameters for steel grade S355.

\begin{tabular}{|c|c|c|c|c|}
\hline \multirow{2}{*}{$\psi$} & \multirow{2}{*}{$\begin{array}{c}\sigma_{H, \text { eff }} \\
{[\mathrm{MPa}]}\end{array}$} & \multirow{2}{*}{$\begin{array}{c}E_{\text {har,eff }} \\
{[\mathrm{GPa}]}\end{array}$} & $\xi_{E, \text { eff }}=E_{T} / E$ & $\xi_{H, \text { eff }}=\sigma_{H, \text { eff }} / f_{y}$ \\
\cline { 4 - 5 } & & & $\mathrm{FEM}$ & $\mathrm{FEM}$ \\
\hline 0.1 & 287.4 & 27.93 & 0.133 & 0.810 \\
\hline 0.2 & 233.9 & 43.97 & 0.209 & 0.659 \\
\hline 0.3 & 189.3 & 57.32 & 0.273 & 0.533 \\
\hline 0.4 & 151.0 & 68.75 & 0.327 & 0.425 \\
\hline 0.5 & 117.6 & 78.74 & 0.375 & 0.331 \\
\hline
\end{tabular}

\section{Buckling curve analytical formulation}

Making use of the equivalent stress-strain model for postwelding steel, derived in the previous section, the upper bound buckling curve formulation of perfectly straight column with material imperfections considered through the standard pattern of postwelding residual stresses from Fig. 1b takes the following nominal format (the relationship is graphically presented in Fig. $2 b$ using dimensionless coordinates):

$$
N_{b, \text { nom }}=\min \left(N_{c r}, N_{c r, e f f}, N_{c r, h a r}\right)
$$

in which $N_{c r}$ is the Euler elastic critical force, and the inelastic critical forces corresponding to a piece-wise equivalent stress-strain relationship given by Eqn. (1) are calculated as 
follows: $N_{c r, e f f}=N_{H, \text { eff }}+\left(E_{T} / E\right) N_{c r}$ is the critical load with residual stresses taken into account, $N_{c r, h a r}=N_{H, h a r}+\left(E_{h a r} / E\right) N_{c r}$ is the critical load referred to the hardening stiffness, $N_{H, \text { eff }}=\sigma_{H, \text { eff }} A$ and $N_{H, \text { har }}=\sigma_{y, \text { red }} A$.

Using the conventional dimensionless coordinates used in Eurocode 3 [1], Eqn. (3) may be presented in the rearranged format:

$$
\chi_{z}=\min \left[\frac{1}{\bar{\lambda}_{z}^{2}}, \xi_{H, e f f}+\frac{\xi_{E, e f f}}{\bar{\lambda}_{z}^{2}},(1-0.001) f_{y}+\frac{0.001}{\bar{\lambda}_{z}^{2}}\right]
$$

It can be seen that the intersection of the Euler's curve for the residual stress free perfectly straight column and that of the perfectly straight column affected by welding residual stresses travels towards larger values of the slenderness ratio when $\psi_{\text {com }}$ increases. The slenderness ratio corresponding to the intersection point is greater than unity and calculated as follows:

$$
\bar{\lambda}_{z, \text { ref }}=\sqrt{\frac{1-\xi_{E, e f f}}{\xi_{H, e f f}}}
$$

The slenderness given by Eqn. (5) is tabulated in Table 3, where $\xi_{H, \text { eff }}, \xi_{E, \text { eff }}$ from Table 2.

While the members are affected by residual stresses in a lower extent, $\xi_{H, \text { eff }} \rightarrow 1$ and $\xi_{E, \text { eff }} \rightarrow 0$, so the modified slenderness $\bar{\lambda}_{z, \text { ref }} \rightarrow \bar{\lambda}_{z}$.

Table 3. Reference slenderness ratio.

\begin{tabular}{|c|c|c|c|c|c|}
\hline$\psi$ & 0.1 & 0.2 & 0.3 & 0.4 & 0.5 \\
\hline $\bar{\lambda}_{z, \text { ref }}$ & 1.035 & 1.095 & 1.168 & 1.258 & 1.374 \\
\hline
\end{tabular}

The real members are affected by material and geometric imperfections. The global effect of imperfections and their interaction during the progressive yielding under applied axial loads on the buckling resistance may be modeled using the semi-probabilistic minima value approach in which the variables $N_{c r}, N_{c r, e f f}$ and $N_{c r, h a r}$ of Eqn. (3) are considered independent random variables following a Weibull distribution. Such a statistical hypothesis of the minima value approach, resulting from the randomization of variables used in Eqn. (3) is called the Merchant-Rankine's-Murzewski approach (M-R-M approach [5]). In the case study presented herein, such approach leads to a three dimensional Weibull minima distribution of the buckling resistance. As a result, the following equation is obtained for the reduction factor $\chi_{z}$ of an imperfect column subjected to residual stresses and initial bow deformations:

$$
\left(\chi_{z}\right)^{-n}=\bar{\lambda}_{z}^{2 n}+\left(\xi_{H, e f f}+\xi_{E, e f f} \bar{\lambda}_{z}^{-2}\right)^{-n}+\left(0.999+0.001 \bar{\lambda}_{z}^{-2}\right)^{-n}
$$

in which the $n$ is the imperfection factor being an inversion of the equivalent Weibull coefficient of variation $u$, i.e. $n=1 / u$. The $u$ parameter plays the same role as the imperfection factor $\alpha$ in the Eurocode 3 [1]. While the values of $u$ and $\alpha$ are higher, the resistance reduction factor $\chi_{z}$ is lower and vice versa. 


\section{Imperfection factor calibration based on FEM simulations}

The results of finite element simulations are presented in [3] for imperfect columns subjected to the residual stress block presented in the previous sections (for five different values of the compressive residual stress ordinate defined as $0.1,0.2,0.3,0.4$ and 0.5 ) and to geometric imperfections according to the lowest buckling mode (for seven different values of the dimensionless geometric amplitude $\overline{e_{0}}=e_{0} / L$, namely $1 / 10000,1 / 5000$, $1 / 4000,1 / 3000,1 / 2000,1 / 1000$ and $1 / 750$, where $L$ is the column length). The smallest geometric imperfections amplitude corresponds to a quasi-straight column while the largest one to the fabrication tolerance level. Nine values of the column slenderness ratio ranging from the slenderness domain $<0.6,1.4>$ (an interval of 0.1 applied) were used, giving all together over 300 FEM results of the buckling resistance simulations for the steel grade S355 as well as for the selected values of column imperfection parameters and slenderness ratio values. It was observed that for each residual stress arrangement, the buckling reduction factors decrease with the increase of $\overline{e_{0}}$ values. Comparing the results for the same $\overline{e_{0}}$ value but with different $\psi_{\text {com }}$ values one can conclude that the buckling reduction factors also decrease for the increase of the compressive residual stress ordinate. Both imperfections, material and geometric have the degrading effect on the column buckling resistance. Their influence on the reduction factor is magnified for the slenderness ratio around $\bar{\lambda}_{z, \text { ref }}$. Similar simulations of residual stress influence on the flexural buckling of welded I-girders were presented also in $[6,7]$. The FEM calculations of compressed elements with the use of concept of equivalent geometric imperfections were described in [8-11].

\subsection{Imperfection parameter for different material and geometric imperfections}

The results of $\chi_{z, F E M}$ presented in [3] are used hereafter to compute the imperfection factor of Eqn. (6), denoted as $n_{F E M}$, from the following equation:

$$
\chi_{z, F E M}=\left[\bar{\lambda}_{z}^{2 n_{F E M}}+\left(\xi_{H, e f f}+\xi_{E, e f f} \bar{\lambda}_{z}^{-2}\right)^{-n_{F E M}}+\left(0.999+0.001 \bar{\lambda}_{z}^{-2}\right)^{-n_{F E M}}\right]^{-1 / n_{F E M}}
$$

Eqn. (7) cannot be solved explicitly for $n_{F E M}$, therefore an iterative procedure of a Newton type need to be applied in order to do so. Results indicate that there are noticeable differences between $n_{F E M}$ for different values of the slenderness ratio.

The following conclusions can be made:

1. Since the practical value of $n_{F E M}$ is not less than 1 and not greater than those around 10 , the large values of $n_{F E M}$ indicate that any value of $n$ in the neighbourhood of 10 may give an acceptable approximation of FEM results from an engineering point of view.

2 . Regarding the practical range of $n_{F E M}$ values, the imperfection factor is generally of a greater value for the slenderness ratio around $\bar{\lambda}=\bar{\lambda}_{\text {ref }}$ then for the tail values of the slenderness ratio.

\subsection{Approximation with use of a slenderness independent imperfection parameter approach}

The imperfection parameter $n$ is calibrated as a slenderness ratio independent factor $n_{\text {opt }}$ with the calibration made using Mathematica function Non-Linear Model Fit. In all cases 
the convergence of the NMinimize method was very good allowing for finding the global minimum of the goal function inside the domain. The results are presented in Table 4.

The results presented in Table 4 prove that the imperfection parameter $n_{\text {opt }}$ decreases with the increase of both imperfection factors, $\bar{e}_{0}$ and $\psi_{c o m}$ (or increase with the equivalent coefficient of variation $u_{o p t}=1 / n_{o p t}$ ). Applying the results given in Table 4, the interpolation function $n_{\text {opt }}\left(\bar{e}_{0}, \psi_{c o m}\right)$ is calibrated with the use of the Mathematica software. The following linear function can be postulated:

$$
n\left(\psi_{\text {com }}, e_{0}\right)=a_{0}+a_{1} \psi_{\text {com }}+a_{2} e_{0}
$$

Parameters $a_{0}, a_{1}$ and $a_{2}$ are determined as equal to: 4.94, -5.82 , and -1039.62 , respectively. Such approximation is not accurate enough, so a non-linear approximation is assumed in the form:

$$
n\left(\psi_{\text {com }}, e_{0}\right)=b_{0}+b_{1} \psi_{\text {com }}+b_{2} e_{0}+b_{3} \frac{1}{e_{0} \psi_{\text {com }}}
$$

with parameters $b_{0}$ to $b_{3}$ determined as: $3.50,-3.86,-351.69,0.00002889$. The graphical interpretation of both approximations are presented in Fig. 3.

Table 4. Imperfection parameter $n_{\text {opt }}$ for $\mathrm{S} 355\left(\psi_{\text {ten }}=1.0\right)$.

\begin{tabular}{|c|c|c|c|c|c|c|c|}
\hline \multirow{2}{*}{$\begin{array}{c}\text { Dimensionless } \\
\text { compressive residual } \\
\text { stress factor } \psi_{\text {com }}\end{array}$} & \multicolumn{6}{|c|}{ Dimensionless amplitude of initial deflection profile $\bar{e}_{0}$} \\
\cline { 2 - 9 } & $1 / 10000$ & $1 / 5000$ & $1 / 4000$ & $1 / 3000$ & $1 / 2000$ & $1 / 1000$ & $1 / 750$ \\
\hline 0.1 & 5.86 & 4.88 & 4.54 & 4.17 & 3.63 & 2.81 & 2.52 \\
\hline 0.2 & 3.93 & 3.51 & 3.38 & 3.16 & 2.86 & 2.35 & 2.15 \\
\hline 0.3 & 2.92 & 2.69 & 2.61 & 2.50 & 2.33 & 2.00 & 1.87 \\
\hline 0.4 & 2.24 & 2.14 & 2.09 & 2.04 & 1.94 & 1.74 & 1.65 \\
\hline 0.5 & 1.84 & 1.77 & 1.76 & 1.72 & 1.67 & 1.54 & 1.49 \\
\hline
\end{tabular}

a)

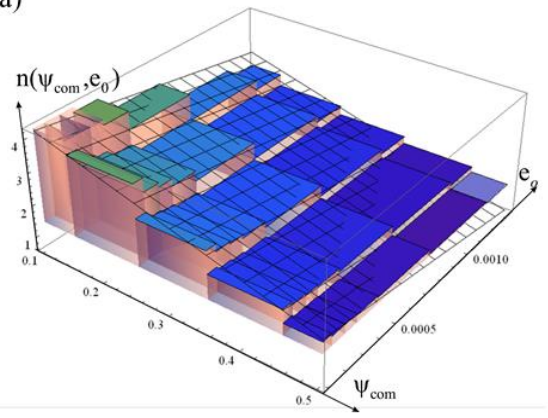

b)

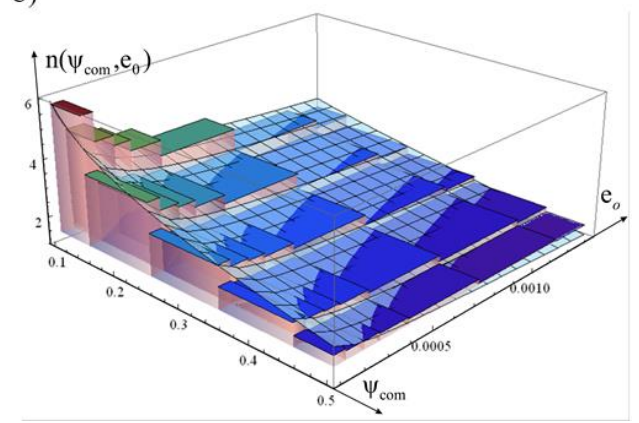

Fig. 3. Linear approximation (a), non-linear approximation of $n_{\text {opt }}$ as a parameter for model described by Eqn. (7) (b).

Fig. 4 presents the results of $\chi_{z}$ from Eqn. (6) based on the analytical formulation and the imperfection parameter $n$ from Eqn. (9), and comparing them with discrete FEM results. Only the extreme cases of $\bar{e}_{0}$ for each $\psi_{c o m}$ values are considered, namely the white circles represent the results corresponding to the lowest geometric imperfection amplitude while 
the black ones to the highest amplitude. The doted curves given in Fig. 4 in colour correspond to function variables in Eqn. (3), $N_{c r}$ in blue, $N_{c r, e f f}$ in red and $N_{c r}$,har in green. From the comparison, the following conclusions can be drawn:

1. While applying a slenderness independent $n$-value, the accuracy level obtained is unevenly distributed over different slenderness ratio ranges.

2. When the slenderness independent imperfection factor $n$ is used, FEM results corresponding to higher geometric imperfection amplitudes may be reproduced more closely by the proposed analytical model than those for the lowest geometric imperfection amplitude.

3. The buckling reduction factor formula given by Eqn. (6) would approximate more closely the FEM results for small geometric imperfections if the imperfection parameter $n$ is a function of the slenderness ratio like in the Eurocode's imperfection parameter $\eta$ is, namely in [1] $\eta=\alpha(\bar{\lambda}-0.2)$.

a)

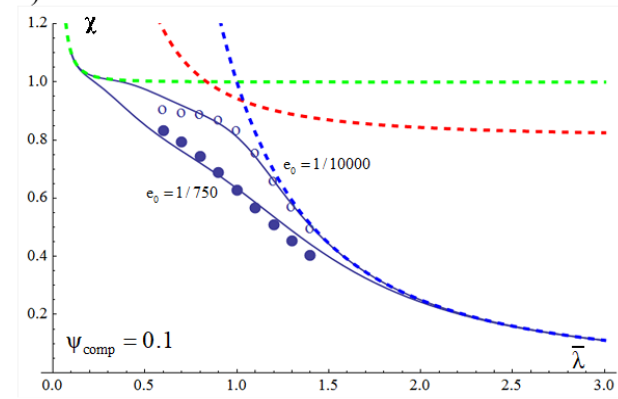

c)

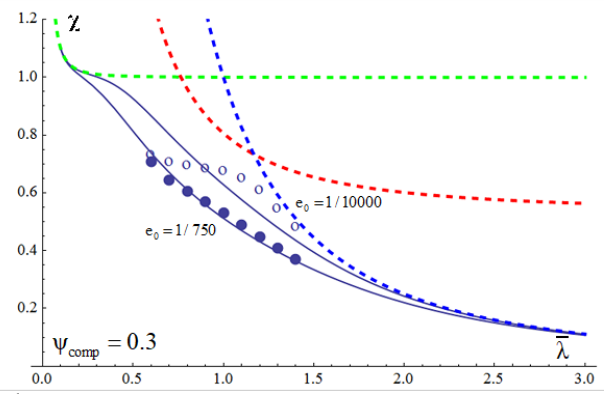

e)

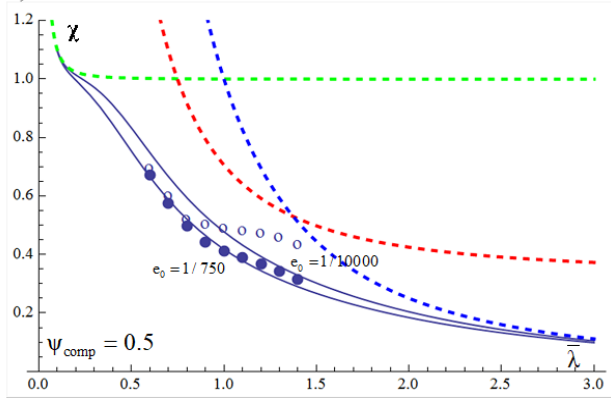

b)

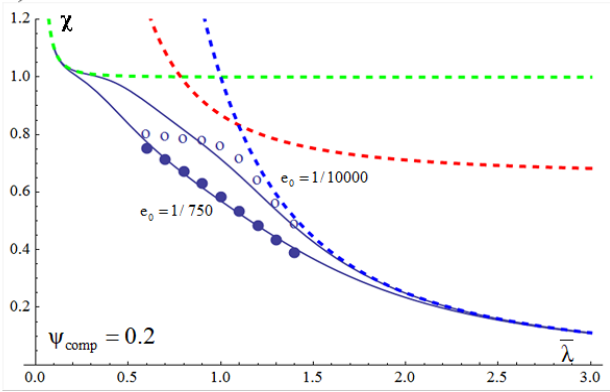

d)

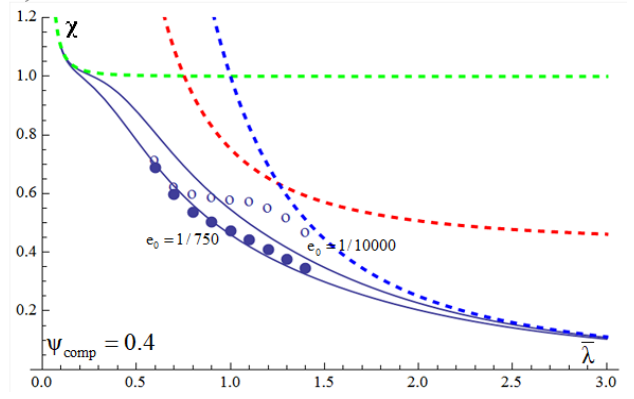

Fig. 4. Reduction factors $\chi_{z}$ calculated analytically and $\chi_{z, F E M}$ from FEM simulations for steel grade S355. 


\section{Summary and conclusions}

The authors presented the issue of buckling resistance modelling of welded I-section columns in a complementary way. The results of FEM calculations were recalled herein and were used to develop the buckling curve analytical formulation. The application of statistical hypothesis of the minima value approach (M-R-M approach) gave a good fit of the analytical solutions and the FEM calculations outcomes. The results of linear and nonlinear approximations were described in detail and summarized in particular subsections. One could observe a higher imperfection factor $\bar{e}_{0}$ triggers a better fit of the analytical solutions and FEM results. The adoption of the very small value of the dimensionless geometric amplitude $\overline{e_{0}}$ equal to $1 / 10000$ leads to the analysis of a practically perfect element. In this case the compliance of results was not satisfactory. The perfect match was observed in case of the value $\overline{e_{0}}$ equal to $1 / 750$.

The results presented in the paper lead to the conclusion that the calculations should be also executed for other types of steel, especially high strength steels. Presently, the authors perform simulations of elements made of steel grade S 690 to check the applicability of M-R-M approach not only in case of common used steels.

\section{References}

1. EN-1993-1-1. Eurocode 3: Design of steel structures Part 1-1: General rules and rules for buildings (Brussels, European Committee for Standardization, 2005)

2. L. Simoes da Silva, R. Simoes, H. Gervasio, Design of Steel Structures, Eurocode 3: Design of Steel Structures, Part 1-1: General Rules and Rules for Buildings (ECCS Eurocode Design Manual, Ernst \& Sohn, Berlin, 2010)

3. B. Launert, R. Szczerba, M. Gajewski, M. Rhode, H. Pasternak, M. Giżejowski, Mat. test., 59, No. 1, 47-56 (2017)

4. J. Murzewski, Reliability of engineering structures (Arkady, Warszawa, 1989) [in Polish]

5. A.M. Barszcz, M.A. Giżejowski, Arch. of Civ. Eng., LII, 1, 59-86 (2006)

6. B. Launert, M. Rhode, A. Kromm, H. Pasternak, T. Kannengiesser, Mat. R. Proc. 2, 109-114 (2016)

7. Z. Kala, J. Valeš, Eng. Str., 134, 37-47 (2017)

8. T. Tankova, L. Marques, L. Simões da Silva, A. Andrade, J. of Constr. St. R., 128, 839-852 (2017)

9. Z. Kala, Arch. of Civ. and Mech. Eng., 15, 1098-1107 (2015)

10. L. Simoes da Silva, L. Marques, C. Rebelo, J. of Constr. St. R., 66, 575-590 (2010)

11. F. Papp, Eng. Str., 106, 124-136 (2016) 